\title{
El diseño estadístico como estrategia de investigación aplicada al estudio de organizaciones solidarias. Caso Cajibío-Cauca
}

\author{
Statistical design as a research strategy applied to the study of \\ solidarity organizations. Cajibío-Cauca case
}

\author{
Wilson Noé Garcés Aguilar ${ }^{a}$ (i) y Lina Juliana Robayo Coral ${ }^{b}$ \\ ${ }^{a}$ Corporación Universitaria Autónoma del Cauca, Colombia \\ ${ }^{b}$ Fundación Universitaria Católica Lumen Gentium Cali, Colombia
}

\begin{abstract}
Resumen. El departamento del Cauca, ubicado en el suroccidente colombiano, dada la diversidad étnica, la riqueza de tradiciones y el amplio acervo cultural e histórico, constituyen un escenario de estudio en el cual la economía social y solidaria nos permite identificar practicas endógenas de desarrollo territorial necesarias de develar en el plano académico. Y por ello, se presenta a continuación un ejercicio académico que busca acercar el diseño estadístico al estudio de organizaciones solidarias en un departamento de vocación rural, en el cual las lógicas solidarias de las organizaciones requieren estrategias de investigación aplicadas a la comprensión de los fenómenos locales para lograr por esta vía generar conocimiento pertinente y ajustado a las realidades regionales. En consecuencia, el articulo pretende visibilizar la importancia de un diseño estadístico pensado como estrategia pedagógica en la clave de investigación formativa para el estudio de organizaciones solidarias en el caso especifico del departamento del Cauca, concretamente en el municipio de Cajibío. Esto para evidenciar como por la vía estadística es posible fundamentar estudios de carácter social y mas aun estudios organizacionales de invaluable valor para los contextos locales.
\end{abstract}

Palabras Claves. Economía solidaria; diseño estadístico; estrategia de investigación; estudios organizacionales.

\begin{abstract}
The department of Cauca, located in southwestern Colombia, given the ethnic diversity, the richness of traditions and the wide cultural and historical heritage, a study scenario in which the social and solidarity economy allows us to identify endogenous practices of territorial development potentially of unveil at the academic level. And therefore, an academic exercise is presented below that seeks to bring statistical design closer to the study of solidarity organizations in a department of rural vocation, in which the solidarity logic of organizations requires research strategies applied to the understanding of local phenomena to achieve in this way generate relevant knowledge and adjusted to regional realities. Consequently, the article aims to make visible the importance of a statistical design thought as a pedagogical strategy in the key of formative research for the study of solidarity organizations in the specific case of the department of Cauca, specifically in the municipality of Cajibío. This to show how by statistical means it is possible to base studies of a social nature and even more organizational studies of invaluable value for local contexts.
\end{abstract}

Keywords. Solidarity economy; statistical design; research strategy; organizational studies. 
Como citar. W. N. Garcés Aguilar y L. J. Robayo Coral, "El diseño estadístico como estrategia de investigación aplicada al estudio de organizaciones solidarias. Caso Cajibío-Cauca", Jou. Cie. Ing, vol. 12, no 1, pp. 191-201, 2020. doi:10.46571/JCI.2020.1.17

Recibido: 28/01/2020 Revisado: 17/03/2020 Aceptado: 21/06/2020

\section{Introducción}

Las organizaciones solidarias en el contexto académico son comprendidas desde la economía social y solidaria, considerándose como emergentes según Razeto [1] "de la economía informal y popular, alternativas de supervivencia donde se privilegia lo asociativo, lo familiar y lo grupal, como medios para alcanzar propósitos económicos logrando expansión y reproducción ampliada" [2] y a su vez también se prioriza aquí las necesidades básicas de la naturaleza humana, no las de el consumidor moderno. [3] Para Coraggio [4] en las organizaciones sociales de la economía solidaria el factor cultural determina "no solo resultados económicos, sino acciones para satisfacer necesidades de los mismos actores, productores o consumidores... pero a diferencia de las capitalistas, su interés fundamental es asegurar una calidad creciente de vida para sus miembros, con un modelo de gestión democrático que no está orientada por la ganancia y la acumulación de capital sin límite" [2] Como principio articulador, las actividades económicas tienen que ser socialmente sostenibles, es decir poseer alto grado de autonomía, estar sujetas a decisiones políticas de la comunidad y estar orientadas a los niveles de auto eficiencia. [5]

En Colombia la economía solidaria es definida por el Estado como agenciadora de desarrollo, no obstante, sus prácticas tienen dos vertientes. La primera centrada en la práctica capitalista y de complementariedad a la banca comercial, esta se ha preocupado por el modelo de desarrollo económico y los programas de educación nacional implementados han generado discriminación, segregación y racialización con respecto a diferentes pueblos en el Departamento del Cauca que presentan cosmovisiones otras con respecto al modelo impuesto. La segunda preocupada por construir la economía "otra" que permita la pervivencia y la vida comunal, buscando soluciones concretas a problemáticas estructurales en comunidades debilitadas y vulnerables. El departamento del Cauca por su diversidad étnica, la riqueza de sus tradiciones y su amplio acervo cultural e histórico, constituyen un escenario de estudio en el cual la economía social y solidaria nos permite identificar practicas endógenas de desarrollo territorial necesarias de develar en el plano académico.

Y por ello, se presenta a continuación un ejercicio académico que busca acercar el diseño estadístico al estudio de organizaciones solidarias en un departamento de vocación rural, en el cual las lógicas solidarias de las organizaciones requieren estrategias de investigación aplicadas a la comprensión de los fenómenos locales para lograr por esta vía generar conocimiento pertinente y ajustado a las realidades regionales. Compartiendo con Montaño [6] que "el avance del estudio de las organizaciones reside precisamente en el reconocimiento de las particularidades institucionales, culturales, etc. que delimitan y orientan nuestra mirada analítica" y por esta vía enriquecen la pertinencia del estudio de las organizaciones, en este caso, solidarias. Además, el estudio de las organizaciones no siempre es perceptible bajo la lógica cotidiana. Ello posibilita la generación de una mejor comprensión y aceptación de la diversidad de los distintos sectores de la organización. [7] En consecuencia, el articulo pretende visibilizar la importancia de un diseño estadístico pensado como estrategia pedagógica en la clave de investigación formativa para el estudio de organizaciones solidarias en el caso especifico del departamento del Cauca, concretamente en el municipio de Cajibío. Esto para evidenciar como por la vía estadística es posible fundamentar estudios de carácter social y mas aun estudios organizacionales de invaluable valor para los contextos locales. 
El diseño estadístico como estrategia de investigación...

\section{Métodos y materiales}

El diseño estadístico [8] implica la identificación de la población objeto de estudio, selección de muestra, diseñar los instrumentos cuantitativos de recolección de información, el procesamiento y análisis de los resultados para finalizar con la fiabilidad y validez de la investigación. Dicho diseño estadístico se soporta para este estudio sobre la delimitación espacial concreta del municipio de Cajibío-Cauca, sin embargo, la población objeto de estudio son las Organizaciones sociales y solidarias (OSS) en contexto de interculturalidad en el departamento del Cauca, Colombia.

\subsection{Establecimiento del marco de muestreo}

Para el establecimiento del marco muestral se parte de la información de la Unidad Administrativa de Organizaciones Solidarias [9] sobre las entidades sin animo de lucro activas en el Cauca hasta marzo de 2017, determinándose como universo 6.187 organizaciones, distribuidas así:

$\begin{array}{ccc}\text { Organizaciones denominadas de economía solidaria } \\ \text { Entidades-Cooperativas } & 838 & (13,5 \%) \\ \text { Fondo de empleados } & 15 & (0,2 \%) \\ \text { Asociaciones Mutuales } & 8 & (0,1 \%)\end{array}$

Organizaciones agrupadas en solidarias de desarrollo

$\begin{array}{ccc}\text { Asociaciones Campesinas } & 371 & (6 \%) \\ \text { Corporaciones } & 329 & (5,3 \%) \\ \text { Fundaciones } & 1384 & (22,4 \%) \\ \text { Demás organizaciones: } & 3242 & (52,4 \%)\end{array}$

Para la determinación del tipo de organización a trabajar se estableció entender las organizaciones solidarias desde la perspectiva de maquina trivial y maquina no trivial de Von Forester [10]

Por maquina trivial podemos asimilar las organizaciones solidarias complementarias al capitalismo pues la entrada, función y salida se demarcan claramente en la lógica lineal del Estado Colombiano, dado que "pese a la variedad de contextos de los ejemplos, el esquema de argumento, lógica y operación es el mismo: debido a la relación invariable, f, entre entrada, x, y salida" [10] (Foerster, 1997)

Por maquina no trivial el símil hacia las organizaciones solidarias de tejido comunal, en tanto la característica esencial "es la desobediencia", es decir "obedece a una voz diferente. Tal vez se podría decir que obedece a su voz interior." [10] (Foerster, 1997), con ello la voz interior se entretejería con el abigarramiento de lo común, construido de forma comunitaria.

Las organizaciones solidarias asimiladas como maquinas no triviales tienen un relacionamiento interior y exterior que determina las salidas, en ese sentido su función opera "el sistema como una máquina dentro de otra máquina" [10] (Foerster, 1997).

En consecuencia [10] (Foerster, 1997) reconoce limitaciones de las máquinas no triviales pero a su vez principios tales como "sintéticamente determinadas ... dependientes de la historia... analíticamente indeterminables ... analíticamente impredecibles" [10] mientras que las organizaciones sociales y solidarias son no triviales y por tanto indeterminables, su abordaje se vuelve impredecible, mas su historia determina unos rasgos ontológicos importantes para observar lo sintético que las determina, en este caso por el Estado Colombiano, pero en la academia por los economistas sociales. Por tanto, se decide trabajar con las asociaciones campesinas asemejándoles a maquinas no triviales de acuerdo con lo argumentado anteriormente. 


\section{Resultados}

\subsection{Diseño de instrumento de recolección de información}

El instrumento utilizado para la recolección de información fue la encuesta, por lo cual se diseñó un formulario con 16 preguntas. Para la definición de las preguntas se partió por reconocer las categorías de análisis desde las teorías inferencias, lográndose identificar que las categorías de análisis para el presente estudio son organización, gestión y administración.

Categorías Inferencia/Indicios

Organización Schvarstein (1998) [11], Daft et al (2011) [13] y Escobar (2016) [10]

Gestión Guillen et al (1970) [14]

Administración Coraggio (2003) [15]

Agrupando las siguientes variables por categoría:

Organización

1. Conformación de la organización:

a) Participación Genero

b) Edad mujeres

c) Edad hombre

d) Nivel de estudio asociados

2. Motivo de asociación

3. Estructura: Junta directiva, Asamblea, Estatutos y Organigrama

Gestión

4. Registro de producción

5. Logros de la organización

6. Desempeño junta directiva

7. Plataforma estratégica y filosófica

8. Canales de distribución Administración

9. El medio ambiente y las Organizaciones

10. Comunicación de los asociados y directivos

11. Convivencia de los asociados con la junta directiva

Una vez identificados las categorías, las teorías de inferencia y las variables, es necesario establecer los supuestos de las categorías y variables que permitirán generar las inferencias a partir de los resultados. De manera tal que el instrumento de recolección de información considera aspectos tales como:

- Información sobre los socios. Se indaga aspectos básicos como: sexo, edad, nivel de escolaridad, proporción de mujeres y hombres de la asociación, a qué tipo de organización social pertenece. Esta información permite caracterizar como tal a las organizaciones.

- Información sobre la organización - asociación. Se averigua sobre la vereda en la cual está, ubicada, actividad agrícola, NIT, la información permite definir el sistema de producción en las organizaciones.

- Categorías de organización y gestión. En este aparte se enfatiza varios aspectos como lo son: estructura jerárquica, decisión o motivo de asociarse, estructuración de la asamblea y la junta directiva, se manejan registros de producción, los estatutos que tan frecuentemente se dan a conocer, fuentes de crédito, logros, forma de comercialización, plataforma estratégica, infraestructura, asistencia técnica, entre otras. 
- Categoría de administración. Comunicación y convivencia. Con el fin de definir métodos o medios de comunicación adecuados en donde nos permite comprender el inmenso valor de la comunicación interna (y sus esquemas) en la operación productiva y en el resultado final de la gestión corporativa. Es necesario evaluar estas variables con el fin de presentar el papel fundamental que desempeña la comunicación intra organizacional en el diseño y ejecución de estos planes.

\subsection{Cálculo del tamaño de la muestra}

Con el ánimo de poder identificar fiabilidad y validez se determino un tamaño de muestra en el municipio de Cajibío - Cauca, por ser esencialmente rural y contar con la diversidad étnica afrodescendiente, indígena y sin identificación étnica, cumpliéndose con ello el contexto de interculturalidad. También se tuvo en cuenta que se encuentran registradas ante Cámara y Comercio un total de 57 organizaciones solidarias de las cuales solo 54 realmente existen. Tomándose así 54 organizaciones como tamaño de muestra se aplica un nivel de confianza de $95 \%$ para determinar el número de organizaciones a encuestar aplicando la fórmula de poblaciones finitas

$$
\begin{gathered}
n=\frac{Z^{2} P Q N}{N E^{2}+Z^{2} P Q} \\
N=\text { Tamaño de la muestra }=54 \\
Z=\text { Nivel de confianza } 95 \%=1,96 \\
e=0,05 \\
p=0,5 \\
q=0,5 \\
n=\frac{Z^{2} P Q N}{N E^{2}+Z^{2} P Q} \\
\frac{1,96^{2} * 0,5 * 0,5 * 54}{54 * 0,05^{2}+1,96^{2} * 0,5 * 0,5} \\
3,8416 * 0,5 * 0,5 * 54 \\
n=\frac{3,8416 * 0,25 * 54}{540025+3,8416 * 0,5 * 0,5} \\
n=\frac{51,8616}{1,0954} \\
n=47,45=48
\end{gathered}
$$

Organizaciones a encuestar.

Para un nivel de confianza de $95 \%$ se aplicó el instrumento a 48 organizaciones en el municipio de Cajibío, Cauca 


\subsection{Aplicación del instrumento}

Para la aplicación de la encuesta se realizo una selección y evaluación de encuestadores en el marco de la interacción entre encuestador y los representantes de las organizaciones previo proceso de formación. Los encuestadores fueron capacitados en tres módulos: Módulo 1 - Fundamentos de investigación. Módulo 2 - Aplicación de instrumentos de recolección de información. Módulo 3 - Presentación de informes de investigación.

\subsection{Procesamiento y análisis de los resultados}

Una vez aplicadas las encuestas, con los resultados se identifico un alfa de Crombach de 0.80 en general, el cual es aceptable para la prueba en general. No obstante, se considero retirar la pregunta 7 del análisis con el animo de lograr un alpha de crombach de 0.8145

Cronbach Alpha and Related Statistics

\begin{tabular}{ccccc}
\hline Items & Cronbach Alpha & Std. Alpha & G6(smc) & Average R \\
\hline All itmes & 0.8032 & 0.8047 & 0.8582 & 0.2048 \\
\hline P1 excluded & 0.8035 & 0.8045 & 0.8509 & 0.2152 \\
\hline P2 excluded & 0.7968 & 0.7979 & 0.846 & 0.2084 \\
\hline P3 excluded & 0.8063 & 0.8082 & 0.8577 & 0.2193 \\
\hline P4 excluded & 0.7925 & 0.794 & 0.8488 & 0.2044 \\
\hline P5 excluded & 0.7856 & 0.7873 & 0.8374 & 0.198 \\
\hline P6 excluded & 0.7868 & 0.7891 & 0.838 & 0.1996 \\
\hline P7 excluded & 0.8145 & 0.8148 & 0.8583 & 0.2268 \\
\hline P8 excluded & 0.7928 & 0.7944 & 0.8463 & 0.2048 \\
\hline P9 excluded & 0.7916 & 0.7932 & 0.8483 & 0.2036 \\
\hline P10 excluded & 0.7878 & 0.789 & 0.8435 & 0.1996 \\
\hline P11 excluded & 0.7922 & 0.7935 & 0.8479 & 0.204 \\
\hline P12 excluded & 0.7885 & 0.7883 & 0.8422 & 0.1989 \\
\hline P13 excluded & 0.779 & 0.7817 & 0.8375 & 0.1927 \\
\hline P14 excluded & 0.7956 & 0.7977 & 0.8505 & 0.2081 \\
\hline P15 excluded & 0.7812 & 0.7842 & 0.8337 & 0.195 \\
\hline P16 excluded & 0.7861 & 0.7878 & 0.8445 & 0.1984 \\
\hline
\end{tabular}

\section{Conclusiones}

\subsection{Correlación}

En términos de la correlación intraclase tenemos como resultado

\begin{tabular}{|c|c|c|c|c|c|c|c|c|c|c|c|c|c|c|c|}
\hline P1 & P2 & P3 & $\mathrm{P} 4$ & P5 & P6 & P7 & P8 & P9 & P10 & P11 & P12 & P13 & P14 & P15 & P16 \\
\hline P1 & 1.000 & & & & & & & & & & & & & & \\
\hline P2 & 0.295 & 1.000 & & & & & & & & & & & & & \\
\hline P3 & -0.076 & 0.130 & 1.000 & & & & & & & & & & & & \\
\hline $\mathrm{P} 4$ & 0.089 & 0.019 & 0.104 & 1.000 & & & & & & & & & & & \\
\hline P5 & 0.012 & 0.221 & 0.042 & 0.295 & 1.000 & & & & & & & & & & \\
\hline P6 & 0.123 & 0.219 & 0.142 & 0.131 & 0.109 & 1.000 & & & & & & & & & \\
\hline P8 & 0.019 & 0.155 & 0.230 & 0.290 & 0.430 & 0.291 & 1.000 & & & & & & & & \\
\hline P9 & -0.010 & 0.240 & 0.224 & 0.105 & 0.266 & 0.434 & 0.263 & 1.000 & & & & & & & \\
\hline P10 & 0.325 & 0.204 & -0.131 & 0.279 & 0.411 & 0.256 & 0.265 & 0.214 & 1.000 & & & & & & \\
\hline P11 & 0.175 & 0.307 & 0.075 & 0.221 & 0.308 & 0.312 & 0.279 & 0.320 & 0.363 & 1.000 & & & & & \\
\hline P12 & 0.159 & 0.360 & 0.120 & 0.258 & 0.320 & 0.108 & 0.291 & 0.256 & 0.365 & 0.267 & 1.000 & & & & \\
\hline P13 & 0.277 & 0.167 & 0.153 & 0.294 & 0.469 & 0.428 & 0.234 & 0.397 & 0.365 & 0.195 & 0.250 & 1.000 & & & \\
\hline P14 & 0.162 & 0.267 & 0.099 & 0.291 & 0.224 & 0.188 & 0.001 & 0.106 & 0.033 & 0.033 & 0.270 & 0.230 & 1.000 & & \\
\hline P15 & 0.141 & 0.039 & 0.207 & 0.360 & 0.261 & 0.544 & 0.205 & 0.334 & 0.192 & 0.016 & 0.296 & 0.542 & 0.436 & 1.000 & \\
\hline P16 & -0.004 & 0.160 & 0.125 & 0.362 & 0.420 & 0.353 & 0.240 & 0.227 & 0.338 & 0.247 & 0.181 & 0.301 & 0.245 & 0.431 & 1.000 \\
\hline
\end{tabular}

Al respecto se puede mencionar la falta de relación entre P1 y P3, P1 y P9, P1 y P16, P3 y $\mathrm{P} 10$, no obstante las relaciones mayores de 0.4 y con un máximo de 0.5 , entre las que encontramos 
las resaltadas en verde en la tabla llama la atención la relación entre canales de comercialización y la comunicación entre los asociados y el representante de la organización con una relación del 0.542

\subsection{Validez}

En términos de validez se realizó un análisis factorial exploratorio a las 16 preguntas resultantes del alpha de crombach.

Los resultados de KMO fueron:

\begin{tabular}{|c|c|c|c|c|c|c|c|c|c|c|c|c|c|c|c|}
\hline $\begin{array}{c}\text { KMO } \\
\mathbf{P 1} \\
\end{array}$ & P2 & P3 & P4 & P5 & P6 & P7 & P8 & P9 & P10 & P11 & P12 & P13 & P14 & P15 & P16 \\
\hline 0.485 & 0.626 & 0.605 & 0.756 & 0.652 & 0.617 & 0.691 & 0.845 & 0.752 & 0.706 & 0.719 & 0.773 & 0.673 & 0.679 & 0.809 & 0.700 \\
\hline
\end{tabular}

Brindando un buen nivel de ajuste. Y un matriz con rotación Varimax así:

$\begin{array}{lccccccc}\text { Factor } & \text { Matrix (rotated Varimax) } & & & & & \\ & \mathbf{1} & \mathbf{2} & \mathbf{3} & \mathbf{4} & \mathbf{5} & \text { Commun } & \text { Specific } \\ \text { P1 } & 0.183 & 0.122 & 0.604 & -0.465 & -0.216 & 0.675 & 0.325 \\ \text { P2 } & 0.110 & -0.010 & -0.025 & -0.835 & -0.102 & 0.721 & 0.279 \\ \text { P3 } & 0.220 & -0.031 & -0.723 & -0.208 & -0.136 & 0.633 & 0.367 \\ \text { P4 } & 0.045 & -0.651 & 0.034 & 0.046 & -0.372 & 0.568 & 0.432 \\ \text { P5 } & 0.109 & -0.762 & 0.019 & -0.175 & -0.037 & 0.625 & 0.375 \\ \text { P6 } & 0.860 & -0.063 & -0.002 & -0.090 & -0.029 & 0.753 & 0.247 \\ \text { P8 } & 0.207 & -0.600 & -0.266 & -0.196 & 0.208 & 0.556 & 0.444 \\ \text { P9 } & 0.650 & -0.166 & -0.251 & -0.265 & 0.140 & 0.603 & 0.397 \\ \text { P10 } & 0.248 & -0.542 & 0.523 & -0.261 & 0.130 & 0.714 & 0.286 \\ \text { P11 } & 0.257 & -0.370 & 0.075 & -0.491 & 0.343 & 0.568 & 0.432 \\ \text { P12 } & 0.002 & -0.421 & -0.044 & -0.572 & -0.240 & 0.565 & 0.435 \\ \text { P13 } & 0.627 & -0.338 & 0.170 & -0.095 & -0.236 & 0.602 & 0.398 \\ \text { P14 } & 0.091 & -0.121 & -0.045 & -0.248 & -0.801 & 0.728 & 0.272 \\ \text { P15 } & 0.642 & -0.262 & -0.037 & 0.098 & -0.568 & 0.814 & 0.186 \\ \text { P16 } & 0.347 & -0.576 & -0.012 & 0.050 & -0.202 & 0.495 & 0.505 \\ & 2.375 & 2.551 & 1.337 & 1.825 & 1.533 & 9.620 & 5.380\end{array}$

Presentándose una matriz con el método de Bartlett así:

\begin{tabular}{lccccc}
\multicolumn{2}{r}{ Factor Scores Matrix - Bartlett's Method } & & & & \\
& $\mathbf{1}$ & $\mathbf{2}$ & $\mathbf{3}$ & $\mathbf{4}$ & $\mathbf{5}$ \\
\cline { 2 - 6 } P1 & 0.071 & 0.229 & 0.461 & -0.248 & -0.126 \\
P2 & -0.043 & 0.166 & -0.104 & -0.624 & -0.042 \\
P3 & 0.038 & 0.030 & -0.522 & -0.155 & -0.027 \\
P4 & -0.137 & -0.299 & 0.008 & 0.102 & -0.151 \\
P5 & -0.119 & -0.383 & -0.028 & 0.006 & 0.034 \\
P6 & 0.576 & 0.224 & 0.026 & 0.038 & 0.172 \\
P8 & -0.013 & -0.249 & -0.188 & -0.037 & 0.157 \\
P9 & 0.249 & 0.048 & -0.174 & -0.094 & 0.181 \\
P10 & 0.031 & -0.274 & 0.428 & -0.029 & 0.169 \\
P11 & 0.053 & -0.096 & 0.000 & -0.183 & 0.221 \\
P12 & -0.142 & -0.135 & -0.075 & -0.235 & -0.093 \\
P13 & 0.180 & -0.033 & 0.115 & 0.050 & -0.027
\end{tabular}




\begin{tabular}{lccccc} 
P14 & -0.165 & 0.025 & -0.050 & -0.156 & -0.596 \\
P15 & 0.348 & -0.012 & 0.007 & 0.286 & -0.436 \\
P16 & 0.017 & -0.188 & -0.010 & 0.096 & -0.027 \\
\hline
\end{tabular}

\subsection{Análisis}

De la muestra de organizaciones un $53 \%$ se dedican a la producción de café orgánico, especial y algunas de ellas con procesos de transformación de la almendra. El 22,2\% se dedican a la producción de cana panelera y transformación en panela en diferentes ofertas. El 14,8\% de estas, se dedican a producción agroecológica, es decir café, cana, especies menores, harina de plátano, ganado lechero y de levante. El $7 \%$ realizan actividades agrícolas, la producción de aguacate, artesanías, espárragos, entre otros. Y un $3 \%$ produce tomate de mesa. Los hallazgos dieron cuenta que la mujer tiene un papel fundamental en las organizaciones, pues representan un $63 \%$ de participación. Esto marcado por el papel de madres cabeza de familia en primera medida quienes destacan por la capacidad de emprendimiento familiar y social, especialmente las mujeres entre los 30 a 60 años de edad. El nivel de escolaridad da cuenta del $4 \%$ de asociados egresados de niveles técnicos, tecnológicos o universitarios pregrados. Mientras, que el $33 \%$ de los asociados tienen bachillerato. El $63 \%$ de los asociados cuenta con estudios de nivel primario. Esto determina en la organización quienes interactúan con el Estado, con los comercializadores, con la cooperación nacional o internacional y quienes lideran proceso al interior de la organización. El interés de asociatividad arrojo que el $52 \%$ se conformaron con el interés de mejorar su calidad de vida, el $34 \%$ manifestó que la decisión que los motivo fue obtener recursos por medio de proyectos, mientras que el $4 \%$ dijo que individualmente no se pudo trabajar por lo que decidieron unir esfuerzos, el $1 \%$ dijo que para ser reconocidos y trabajar por los derechos de los desplazados y las minorías. Las formas de organización y administración en la dimensión formal y legal son diversas, por ejemplo, se administra de forma directa mediante junta directiva o asamblea general, pocos lo hacen por medio de administradores, según la encuesta refleja que $81 \%$ dice que se encuentran bien estructuradas. Es decir, cuentan con formas organizativas formales, se reúnen periódicamente en sus reuniones, llevan actas, socializan todo lo referente a proyectos, acuerdos, entre otros asuntos. Mientras que el $19 \%$ manifestó que no esta bien estructurada, es decir que estas son asociaciones semi estructurada, ya que no se realizan ciertos procesos como organización. En pocas palabras no cumplen no realizan algunas actividades en este perfil, aduciendo que la legalidad es del Estado y su económica capitalista. En cuanto a la participación el $75 \%$ de estas organizaciones dice que hay participación en la asamblea general, cuentan con socios activos que participan en las reuniones, referencian sus puntos de vistas según el tema de las reuniones y buscan construir y guiar la organización, mientras que el $25 \%$ reconoce que no existe una asamblea o participación activa, dado que los fines de las organizaciones son temporales y depende de la época del año y los temas a tratar. Considerando que las organizaciones son registradas ante la Cámara y Comercio del Cauca como entidades sin ánimo de lucro y están regidas bajo parámetros mínimos legales, se le preguntó al respecto y el $52 \%$ afirmó que cuentan con el organigrama ya diseñado de tipo horizontal, en tanto el $33 \%$ de las organizaciones señalo no tener un organigrama, mientras el $15 \%$ no respondió esta pregunta por falta de conocimiento. Al conversar más a fondo sobre el tema, se halló que no existe una jerarquización en práctica en las organizaciones, solo de papel, incluso quienes no conocen o no tienen llevan prácticas de democracia ampliada, en la cual todas las personas lograr debatir y llegar a un consenso, o en su defecto a asumir la responsabilidad de los daños que cause una decisión sobre el colectivo. Entre los logros alcanzados y que resaltan las organizaciones tenemos que el $50 \%$ de estas realizaron; creación de un fondo rotatorio que surge por las cuotas de afiliación de cada asociado, de la misma manera que algunas de estas se beneficiaron mediante proyectos productivos, otro logro sustancial ha sido conformar como tal la asociación y permanecer en el tiempo, manifestaron el compromiso y la sana convivencia 
entre los asociados. El $25 \%$ dijo que sus logros se afirmaron en el acceso a ciertos proyectos, el cual les permitió adquirir maquinaria, fortalecer su fondo rotatorio hasta el punto de sostenerse y servir como entidad crediticia para los mismos asociados. El $10 \%$ manifestó que su logro es obtener su propio terreno y su edificación. Mientras que el $7 \%$ de las organizaciones dijeron que su logro es la adquisición de maquinaria para realizar sus procesos productivos con eficiencia. El $8 \%$ se limito a responder. De lo anterior se puede deducir que estas organizaciones tienen una forma de administración que permite una participación ampliada en las labores administrativas y productivas. Respecto a su estructura se puede plantear la funcionalidad de esta, en términos de no concentración de poder, pero si asignación de responsabilidades de manera rotativa. Así mismo la participación por parte de las instancias de decisión como las asambleas generales es activa, en casi todas las actividades que se realizan aun cuando la participación de los socios es más del $70 \%$, por lo que se deduce que hay participación, interés y compromiso. $\mathrm{Al}$ preguntar por las cuentas, se menciona que, por el carácter agrícola, se lleva registros por asociado, es decir se detalla la producción, costos y gastos por finca-propietario, para tratar de tener datos exactos en el momento de la contribución de cuotas que permitan una redistribución igualitaria. Sin embargo, no se realizan balances por y para la organización. Respecto a la tenencia de maquinaria, se encuentra que el $75 \%$ de total de las organizaciones no cuentan con la maquinaria adecuada, mientras que el $15 \%$ tiene la maquinaria para realizar algunos de sus procesos productivos. La disponibilidad de maquinaria de estas organizaciones esta orientada a la producción de café y cana, por lo que se pudo establecer que cuentan con despulpadoras, secadoras, para el lavo del café fundamentalmente, mientras que para la producción de panela existe lo necesario, como trapiches. Esto limita la eficiencia de la producción debido a que todos los asociados no tienen la disponibilidad y los medios para acercarse a quienes las tienen. De las organizaciones entrevistadas, solo 7 manifestaron que si tenían la maquinaria y equipo necesarios para realizar sus procesos productivos completos. También se evaluó el estado de la infraestructura, teniendo en cuenta que son pocas organizaciones que cuentan edificaciones para almacenar, realizar ciertos procesos del café y la cana, solo el $6 \%$ al menos tiene una edificación casi con las condiciones mínimas requeridas por sanidad. De otro lado el $90 \%$ no cuenta con edificaciones propias y en poco caso se arrienda, lo que genera un sobrecosto a cada asociado que deba hacer llegar su producto a la poca maquinaria disponible. En cuanto a los canales de comercialización, se identifico que el $63 \%$ de las organizaciones comercializan por medio de intermediarios, especialmente los cafetos que producen solo almendra, es decir café especial u orgánico sin ninguna transformación, en el que juega un papel monopólico la Federación de Cafeteros. También se encuentran los que producen panela, especies menores, ganado entre otros, que les implica de acuerdo con el producto cosechado, se comercializa en tiendas, plazas de mercado, centros de acopio e incluso los mismos asociados. En este panorama, se puede concluir que se tienen unas precarias maneras de funcionamiento que hacen al campesinado de Cajibío propenso a los paquetes tecnológicos que se ofrecen por parte de los monopolios de comercio, pues hay un gran numero que no esta tecnificado, no tiene la disponibilidad de maquinaria, en cuanto a su infraestructura solo algunas cumplen las mínimas condiciones de sanidad para sus procesos, no se tiene acceso claros y posibles a un mercado el cual les permita ofertar sus productos a un precio que ellos mismos establezcan.

\subsection{Reflexión}

El presente articulo espera ser una fuente de consulta y enseñanza del diseño estadístico para estudiantes pregraduales y posgraduales en los seminarios de estudios cuantitativos o estadística para la investigación en el contexto del departamento del Cauca. En tanto, muestra paso a paso un ejercicio real devenido de una tesis doctoral, que sirvió para argumentar el contexto de las organizaciones solidarias y en consecuencia refleja la construcción de un caso de estudio en el que se realizo trabajo de campo por un semestre. A manera de reflexión será necesario 
plantear que las organizaciones rurales responden a la capacidad de resistencia en la práctica de quienes a pesar de un panorama desolador hacen de la finca un espacio de libertad. Pues de acuerdo a Pérez [16] la construcción concreta deviene de la construcción mental colectiva que determina la existencia y por tanto el proyecto de transformación desde la base comunal. De acuerdo con Alvarado [17] estas prácticas más que simples prácticas de producción o gestión; resultan ser complejos proceso de subjetivación política, que se dan en medio de territorialidades para afirmarse de manera consciente con su autonomía política, económica y cultural. En esa medida habría que discutir el sentido de las organizaciones sociales en contexto de resistencia, subsistencia y pervivencia. En las cuales la administración vuelve a la esencia de la vida y propone el autogobierno como punto de partida, la razón comunal como espectro de convivencia y el bien común como proceso, pero también como fin, llamado de atención realizado por Boissin [18] en cuanto fragilidad del estatus epistemológico de las teorías de gestión estratégica.

Por ejemplo en el caso de estudio expuesto la gestión solo aparece para ellos con la intervención del Estado, mientras que las organizaciones sociales y solidarias se autogestionan o gestan con fines específicos, mientras que lo comunitaria se concertan y por tanto no se gestiona (lo no trivial), lo cual da cuenta de una proliferación no considerada por Chanlat [19] y que hace sentido cuando se identifica el abigarramiento de lo comunal con las organizaciones sociales y solidarias y los pliegos de peticiones o negociaciones con el Estado.

De ahí que pretender desarrollar pensamiento administrativo implicaría según Bourdieu "examinar a las teorías y los métodos en su aplicación para determinar que hacen con los objetos y que objetos hacen" [20]. Para con ello brindar pues una nueva entrada a las teorías de la organización y escapar por tanto de la maquina trivial que deja entrever [21] la cual se constituye en autopoiética metafóricamente, pues la teoría " nueva" de la administración termina replicando en el fondo las narrativas con-coloniales.

\section{Agradecimientos}

El autor principal agradece a la Corporación Universitaria Autónoma del Cauca por la comisión de estudios concedida y al proyecto de formación del talento humano "Innovación Cauca" de la Universidad del Cauca por la beca doctoral concedida a través de la convocatoria de Doctorados Nacionales 2016 Así mismo, al Doctorado en Administración de la Universidad del Valle, el presente documento es resultado del proceso de formación y es requisito para la finalización de los estudios.

\section{References}

[1] L. Razeto (1997). Los caminos de la economía de solidaridad. Buenos Aires: Ediciones Lumen-Humanitas.

[2] M. Riascos (2014). Gestión innovadora en una organización de economía solidaria, estudio de caso. Memorias Encuentro Internacional de Investigadores en Administración 2014

[3] L. Razeto (2009). "El Debate Sobre Las Necesidades, y La Cuestión de La 'Naturaleza Humana."” Polis (07176554), vol. 8, no. 23, May 2009, pp. 139 167. EBSCOhost, search.ebscohost.com/login.aspx? direct $=$ true\&db=fua\& $A N=47525344 \& l$ ang $=$ es\&site $=$ ehost - live.

[4] J. L. Coraggio (2011). Economía social y solidaria. El trabajo antes que el capital. Quito: Ediciones AbyaYala.

[5] M. Loreto Alarcón-Rodríguez, M. Delpino Chamy, S. Valeska Fernández-Castillo, J. Soto-Abarzúa (2019). Prácticas Agroecológicas en Territorios Rururbanos Del Área Metropolitana De Concepción: Aportes Desde La Economía Social Y Solidaria a La Sustentabilidad Urbana. Urbano, 22(39), 42-63 Doi:10.22320/07183607.2019.22.39.03

[6] L. Montaño Hirose (2006), "La Sociología de las Organizaciones: desarrollo, rompimientos y perspectivas", en Enrique de la Garza Toledo (coord.), Tratado latinoamericano de sociología, Anthropos y Universidad Autónoma Metropolitana-Iztapalapa, Barcelona, pp. 195-208.

[7] L. Montaño Hirose, M. Rendón Cobián (2015). La Intervención Organizacional Como Dispositivo De Dilucidación De La Responsabilidad Social. Gestión y Estrategia, [s. 1.], n. 48, p. 25-38.

[8] Webster, L.Allen (2000). Estadística Aplicada a los Negocios y la Economía. (3a Ed.). Bogotá: Irwin McGrawHill. 
[9] UAOS (Unidad Administrativa de Organizaciones Solidarias) (2016) Estadísticas RUES. Link http://www.orgsolidarias.gov.co/planeaci\%C3\%B3n-y-control/planes-y-programas/ ruesVisitada20deDiciembrede2016

[10] H. V. Foerster (1997). Principios de autoorganización en un contexto socioadministrativo. Cuadernos de Economía, XVI No 26, 131-162.

[11] L. Schvarstein, (1998). Diseño de Organizaciones: Tensiones y paradojas. Buenos Aires: Paidós. 424 p.

[12] R. L. R. L. Daft, M. Rodriguez, D. Rodríguez, A. B. F. Cortagerena, C. B. Cortagerena, J. A. Stoner, G. R. G. Jones (2011). Teoría y diseño organizacional (No. 65.014). Cengage Learning.

[13] Arturo Escobar (2016). Diseño y autonomía. Editorial Universidad del Cauca, Popayán.

[14] A. Guillén, E. Y. Yacuzzi, J. S. Selser, S. L. Lazzati, P. H. Hermel, L. Riveros, (1970). Democracia directa: autogestión y socialismo (No. 330.852). OIT.

[15] J. L. Coraggio (2003) La Economía Social como vía para otro desarrollo social, artículo central del debate "Distintas propuestas de Economía Social" lanzado en Urbared, Red de políticas sociales urbanas, proyecto conjunto de la UNGS (Argentina) y la UNAM (México), en www.urbared.ungs.edu.ar, publicado en Pobreza Urbana y Desarrollo (Serie FORTAL), IIED-AL, Número 1.

[16] R. Perez 1998, difussion mai 1999. Le choc des paradigmes en Sciences de Gestion. Unpublished manuscript. Paris.

[17] Alavarado Torres, M. Territorialidades campesinas en Loja, Ecuador: análisis de sus dinámicas organizativas a partir de tres casos. Eutopía: Revista de Desarrollo Económico Territorial, [s. 1.], n. 13, p. 89-113, 2018. DOI: 10.17141/eutopia.13.2018.3301

[18] J.-P. Boissin, J.-C. Castagnos, G. Guieu, 2003. Análisis bibliométrico de la teoría de gestión estratégica basada en recursos y competencias. Innovar, Revista de ciencias administrativas y sociales (21), 71-80.

[19] A. Chanlat, 2004. Gestiones y humanismos: una arqueología de la gestión., Iztapalapa.

[20] P. Bourdieu, J.-C. Chamboredon, J.-C Passeron, 1978. El oficio de sociologo, Mexico, Siglo XXI editores.

[21] Jeffrey Pfeffer, (2000): Nuevos Rumbos en la Teoría de la Organización. Oxford University Press México, S.A. de C.V. Págs. Prefacio, Capítulos 1, 8 y 9.
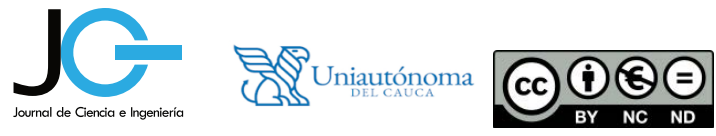